\title{
Accurate Flux Calculations Using Thermographic IR cameras in Concentrated Solar Power Fields
}

\author{
A. Eitan*, G. Naor*, R. Hayut*, I. Segev*, J. Golbert**, S. Pekarsky*, A. Zisken*, G. Medan*, A. Feigelstock*, N. \\ Goldberg* and G. Kroyzer* \\ ${ }^{*}$ Control, Modeling and SFINCS Department, BrightSource Industries Israel, 11 Kiryat Mada St. P.O.Box 45220 \\ Jerusalem 91450, Israel, aeitan@BrightSourceEnergy.com \\ ** Engineering Department, BrightSource Industries Israel, Israel
}

\section{Abstract}

As part of the solar field control system, BrightSource uses thermographic IR cameras that monitor the receiver temperature. The IR cameras are located outdoors in the solar field. By measuring receiver temperature, the accurate radiative flux per area of the receiver is calculated [1] along with the total input flux of the solar field. This paper describes the method of extracting the flux calculations from the IR temperatures maps. The paper also discusses the work done on obtaining accurate readings from the cameras.

\section{Introduction}

BrightSource is a leading company in the CSP (Concentrated Solar Power) field of solar energy. This article will provide some examples of the uses and improvements Brightsource made in the IR measurement system in its last couple of years.

BrightSource uses the "solar power tower" technology (figure 1). Tens of thousands of heliostat mirrors reflect solar beams onto the top of a central tower to heat the water contained in the tower. The receiver in the solar field is located at the top of a tower at a height of about 100-200 meters high. The receiver has several faces. Each of the receiver's faces is made of vertical pipes in which the steam flows.

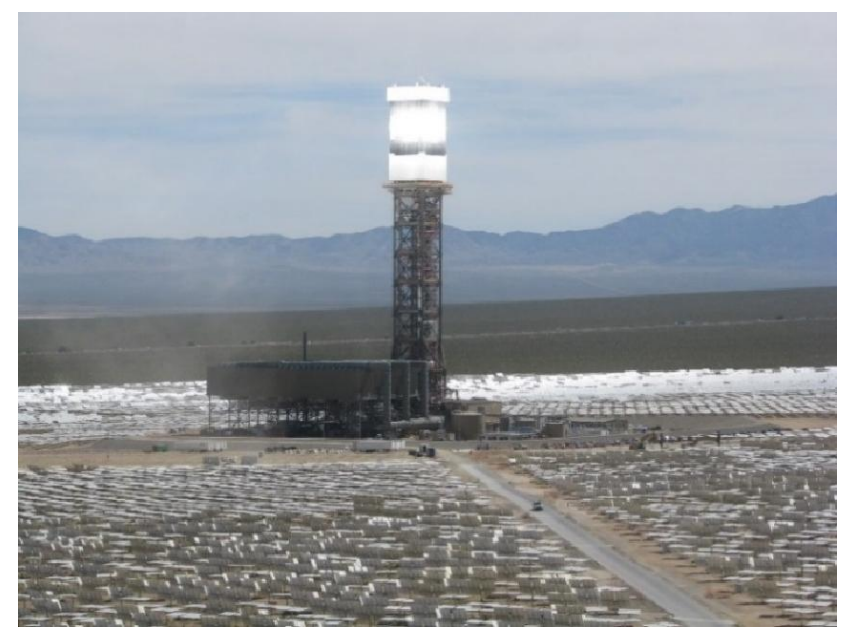

Fig 1. Power Tower during Operation

Unlike traditional boilers in power plants, in the concentrated solar field the energy source comes from the outside, i.e., the sun beams from the heliostats. The sun flux is absorbed by the pipes, coated with a high absorptivity paint, and transferred through the pipe wall to the steam.

The uncooled, bolometric, thermographic IR cameras, used as part of the SFINCS (Solar Field Integrate Control System), are located in the solar field a few hundred meters away from the receiver. The accuracy of these cameras is vital for the maximal utilization of the solar field. This accuracy must be maintained, in spite of harsh conditions such as sizable distances from targets, and variable atmospheric conditions.

Based on temperature maps received from the cameras, applied flux is calculated using heat transfer calculations [1] from the face of the receiver to the steam inside the pipes. This calculated flux verifies the expected flux requested by the SFINCS, and warns about flux density limits that have been exceeded. 


\section{Thermodynamic model}

In order to extract the actual applied sun flux on the receiver from the temperature maps, a full model of the heat transfer needs to be modeled. The first important factor is the absorptivity of the coating for the solar spectrum. The absorbed energy can then transferred by the following three heat transfer methods.

- Convection is computed based on the weather data collected by the control system (ambient temperature and wind speed)

- $\quad$ Radiation is calculated based on the emissivity of the paint of the receiver

- $\quad$ Conduction, the net heat transfer rate, is calculated using equation (1).

The net heat transfer rate $(\mathrm{Q})$ calculation is based on three factors: the temperature of the skin measured by the IR camera (see figure 4), the temperature of the steam inside the pipes (measured by thermocouples spread in many places inside the pipes) and the total heat resistance of pipe wall.

$$
Q=\frac{T_{\text {Skin }}-T_{\text {Bulk }}}{R}
$$

The heat resistance is calculated from the three heat transfers occurring in the pipe wall. See equation (2).

$$
R\left[\frac{m^{2} K}{W}\right]=R_{\text {paint }}+R_{\text {Pipes }}+\frac{1}{h}
$$

The first resistance $\left(R_{\text {Paint }}\right)$ is the resistance of the paint that is applied on the pipes from the outside. This paint has a high absorptivity in the solar emission spectrum and is about 100 microns thick. The paint resistance is measured in a lab. The second resistance (RPipes) is that of the pipes walls. Since the pipe wall material and geometry is known, it is relatively easy to simulate its resistance. See simulation example in figure 2 . The last argument in equation (2) is the heat transfer coefficient $(\mathrm{h})$ at the pipe to steam heat transfer. This is simulated based on known data such as the pipes inner diameter and on data obtained by the sensors that are part of the receivers control system. This sensor data includes: the fluid temperature, which also affect fluid properties (such as viscosity, specific heat, thermal conductivity and density) and fluid velocity.

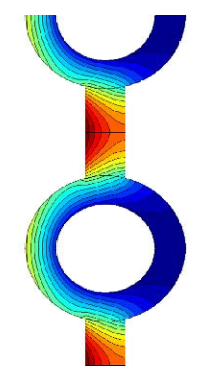

Fig 2. Receiver's pipe heat transfer simulation

After all resistances are calculated, the net heat transfer rate can be found. By knowing the absorptivity of the paint on the pipes and by matching, as in equation (3), the heat transfer rate to the heat applied on the receiver's surface, the flux can be extracted.

$$
Q+\text { Radiation }+ \text { Convection }=\text { Flux } * \text { absorptivity }
$$

A summary of all previous calculations is shown in figure 3, an example of an IR image is shown in figure 4, and the output of the calculation is seen in figure 5. 


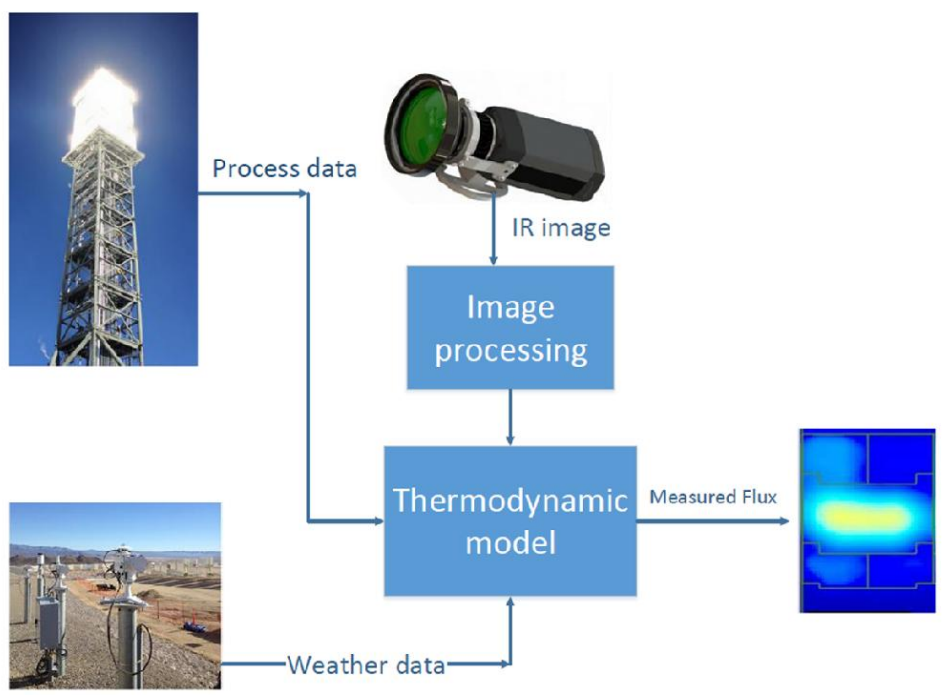

Fig 3. Flux calculation process

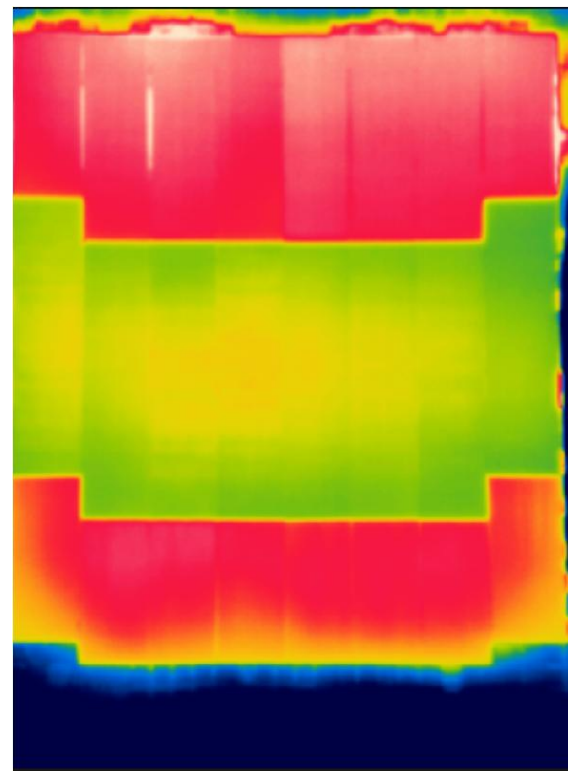

Fig 4. Receiver's Face Temperature Map

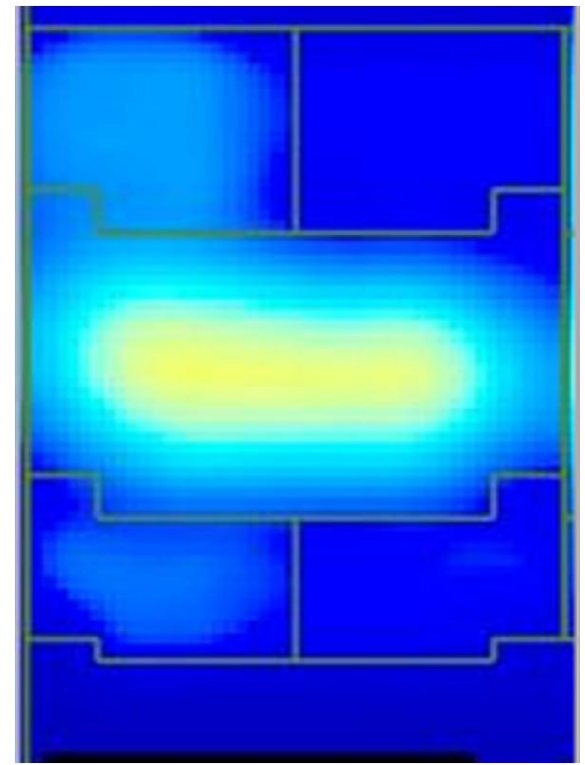

Fig 5. Calculated Flux Map [KW $\left.\mathrm{KW}^{2}\right]$ 


\section{Errors of measurement in current IR system [2]}

The errors apparent in the solar field's IR system result from the camera setup and locations while observing the receiver from a far.

The cameras are situated in a stationary location more than $200 \mathrm{~m}$ away from the receiver center. The cameras are placed in a sealed enclosure with a Germanium window to protect them from the elements. An active cooling unit is attached to the enclosure to maintain the cameras at a constant temperature.

This setup may contribute factors that lead to errors in the temperature measurement. The following discussion outlines these errors, starting from the receiver to the within the camera.

The first factor is the emissivity of the paint applied on the tubes of the receiver. There is an error of about $\pm 3 \%$ in the initial measurement performed by an Emissometer. This error comes in addition to the emissivity variation resulting from the tubes shape (see Figure 2). A change over time as the paint deteriorates may also occur.

The second factor is the Atmospheric optical transmission. The infrared radiation has to travel over more than 200 meters before reaching the camera. The radiation is both scattered and absorbed. Most of the time, the main influence is caused by water vapor that absorbs the radiation. It is important to emphasize that the IR absorption is dependent on the actual volume of water vapor in the air (absolute humidity) since the water capacity in the air is very much temperature dependent, especially at higher temperatures (see figure 6), the humidity error correlates closely with the ambient temperature error. By itself, the ambient temperature negligibly affects the measurement due to the high object temperatures. This water vapor influence is calculated by a LOWTRAN model within the camera. Another factor such as reflected radiation is similar to the issue of ambient temperature; that is, less dominant in our system, due to the high temperatures of the observed object (450K-700K).

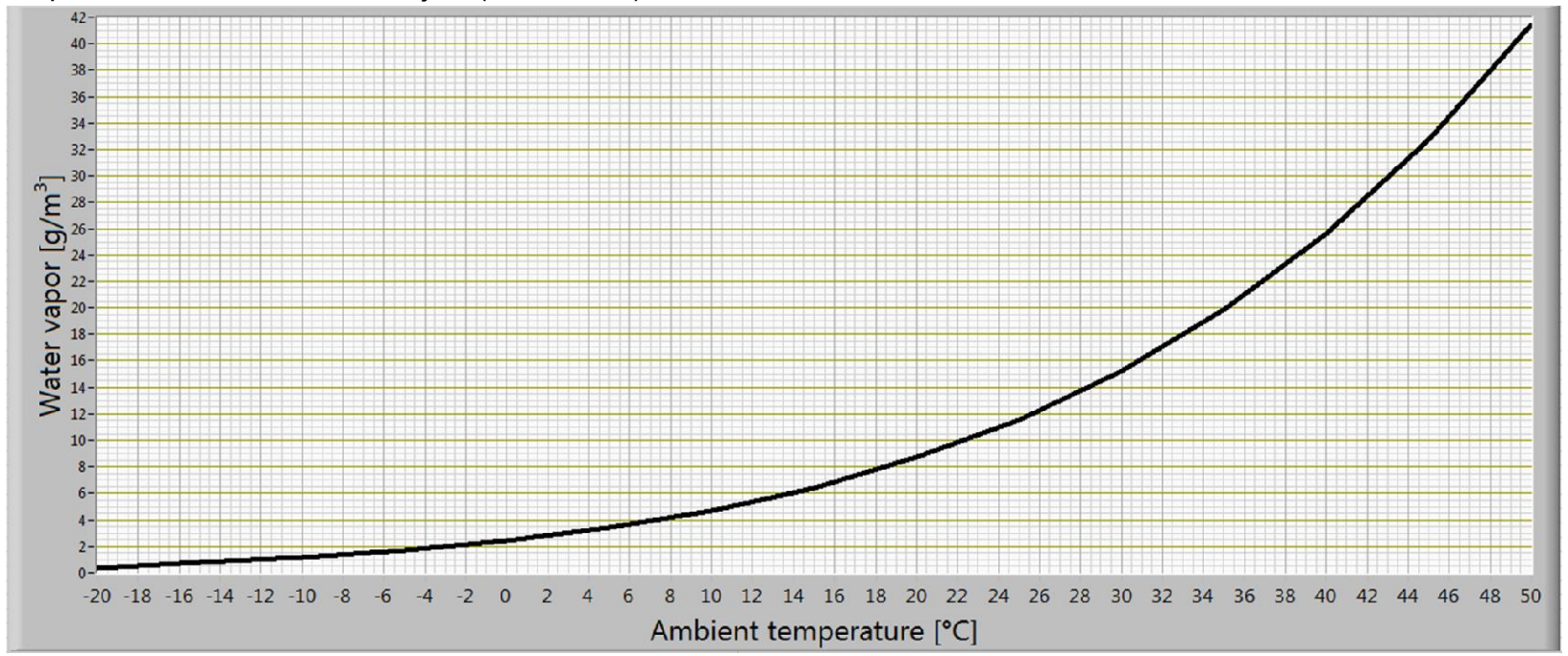

Fig 6. Water vapor in a cubic meter at $50 \%$ relative humidity and different ambient temperatures

After passing through the atmosphere, the IR radiation has to pass through the Germanium window before reaching the camera's optics that have a transmission ratio known to the camera manufacturer and taken into account when the camera is calibrated by the manufacturer.

Finally, the camera subtracts all IR radiation emitted by the camera itself, i.e. the ambient drift compensation. The camera is calibrated at the factory at certain steps of ambient temperatures. In real life, the ambient temperature may vary quickly resulting in an uneven heat distribution inside the enclosure and camera. This can result, in a situation where the values stored in the camera's calibration tables may vary from the actual internal emitted radiation.

Sun radiation reflecting from the receiver surface was calculated to be negligible [3]. Due to the high emissivity of the surface direct sun bouncing off the receiver can add less than a $0.2 \mathrm{~K}$ uncertainty. The other concern is the sun reflected from the heliostats and concentrated on the receiver. This factor is also negligible mostly due to the absorption by the heliostats' glass at wavelengths longer than $3 \mu$. The radiation reflected by the heliostats can add up to $1 \mathrm{~K}$ of uncertainty during the time when the receiver itself is at the higher range of temperatures.

It is important to mention that, as part of solar field control, the following parameters are monitored by calibrated sensors:

- Ambient temperature 
- Relative humidity

- Atmospheric pressure.

- Aerosols attenuation in the visual and NIR range.

- $\quad$ The sun DNI (Direct Normal Irradiance)

\section{Calibration method}

To minimize the errors mentioned in section 3, a calibration method had to be developed. In this method, the receiver becomes one large black body in a known temperature, making it possible to estimate all the error parameters.

First the window's optical transmission factor was found. This was accomplished by measuring a receiver at a constant temperature. With the window in place or out of place, the window's optical transmission parameter is adjusted so that the measured receiver temperature remains the same. After the window optical transmission was found for each camera, the emissivity and atmospheric transmission were calibrated.

There are several receiver operation modes, only one of which is suitable for this calibration. The requirements for the suitable operation mode are:

- No flux can be applied on the receiver during calibration, since then the inner and out temperatures will not match

- High temperature steam must be circulated on the panels during the calibration to stabilize the panel? temperature

A very specific mode was defined that suits all calibration needs. At the end of each day, the steam from the drum is circulated for several minutes after the flux is removed. At that time the large drum reservoir, along with the high steam flow on the "steam generator" part of the receiver, accomplishes a stable panel temperature while the inner and outer panel areas are at the same temperature. At that specific time, the temperatures reported by the thermocouples at the exit of the steam generator equal the panel's outer temperature measured by the IR camera.

The calibration test was repeated at the end of most days. By detecting strange behaviors during the calibration process, and correlating them with different parameters, the accuracy of the camera improved. Since there are more than one solar fields, and each solar field has a receiver with several faces with a camera observing the face, each test includes multiple cameras. This adds to the statistical robustness of the calibration.

Each day's errors are calculated as transmission changes. This is done by finding the transmission added to the base transmission of the window that balances the process thermocouples readings and the IR thermographic reading. Figure 7 provides an example of data collected from one of the solar fields over a month. In this figure the actual temperature error is correlated with the absolute humidity.

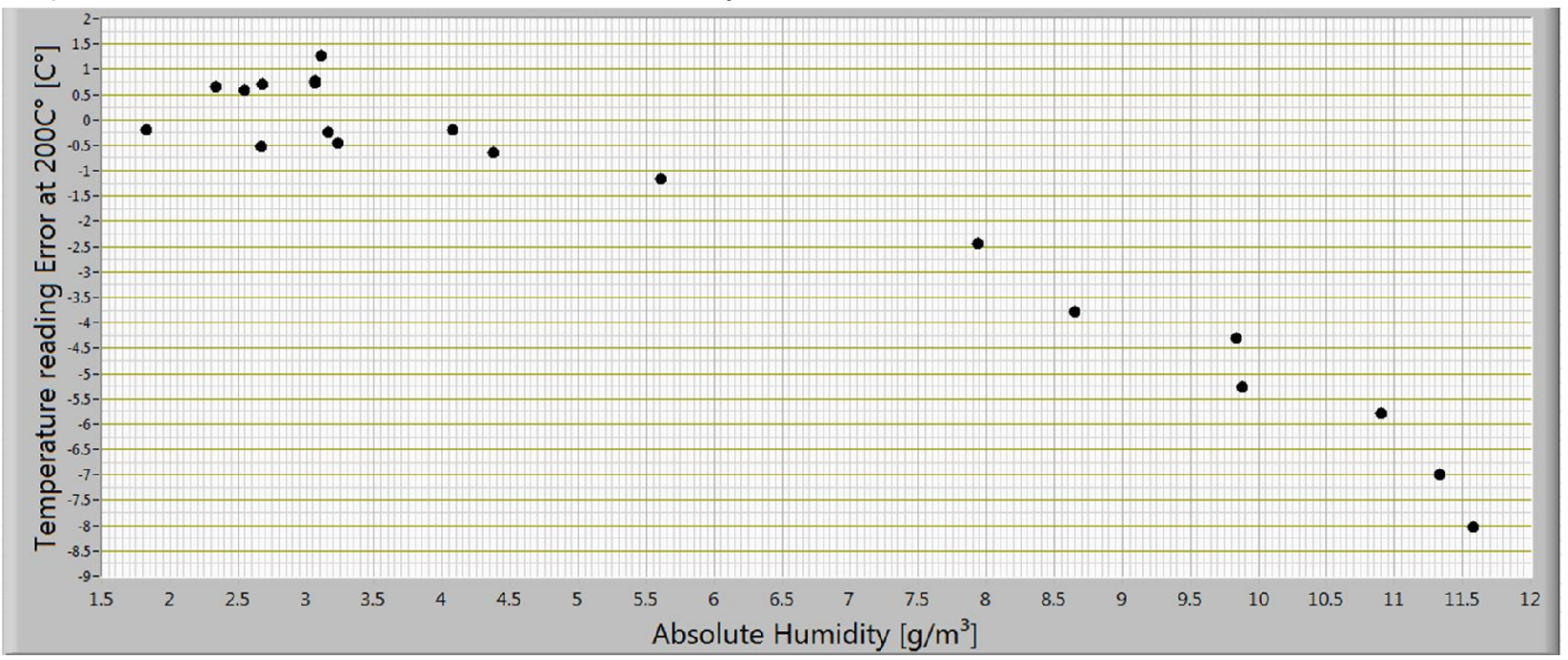

Fig 7. Empirical data of transmission as function of absolute humidity

Note that since the emissivity and the transmission are multiplicative the calibration also contains within it the emissivity correction. 


\section{Error corrections}

During the last couple of years, data was collected to improve the IR system accuracy. The assumptions validated in one site were implemented and verified in a different location that has different weather conditions. During that time, first and second order of magnitude error corrections were found.

Although the camera has an internal atmospheric transmission correction based on a LOWTRAN model, when correlating the atmospheric transmission we got from the camera into empirical data, the curve of the atmospheric transmission (shown previously in figure 7) was slightly different from the model. The new curve parameters were then used as part of the image processing carried out by the control software on a server.

Another error source was found in the camera's temperature. The error is smaller and to show it, a spread of eight different cameras at different dates are plotted in figure 8. Each data point represents a camera with a specific temperature, measured at the aperture of the camera. Near each point there is also the day's number starting from the first day of the test. This was added to verify that changes did not drift over time. As can be seen in figure 8 , there is a slight dependency on the camera's temperature. This temperature is influenced by the combination of both the weather outside the enclosure, and the active cooling unit work. As a result of this finding, the allowed hysteresis of the temperature stabilization unit was reduced; and the temperature within the camera enclosure was stabilized around a fixed temperature, and not allowed to reach the full temperature spec.

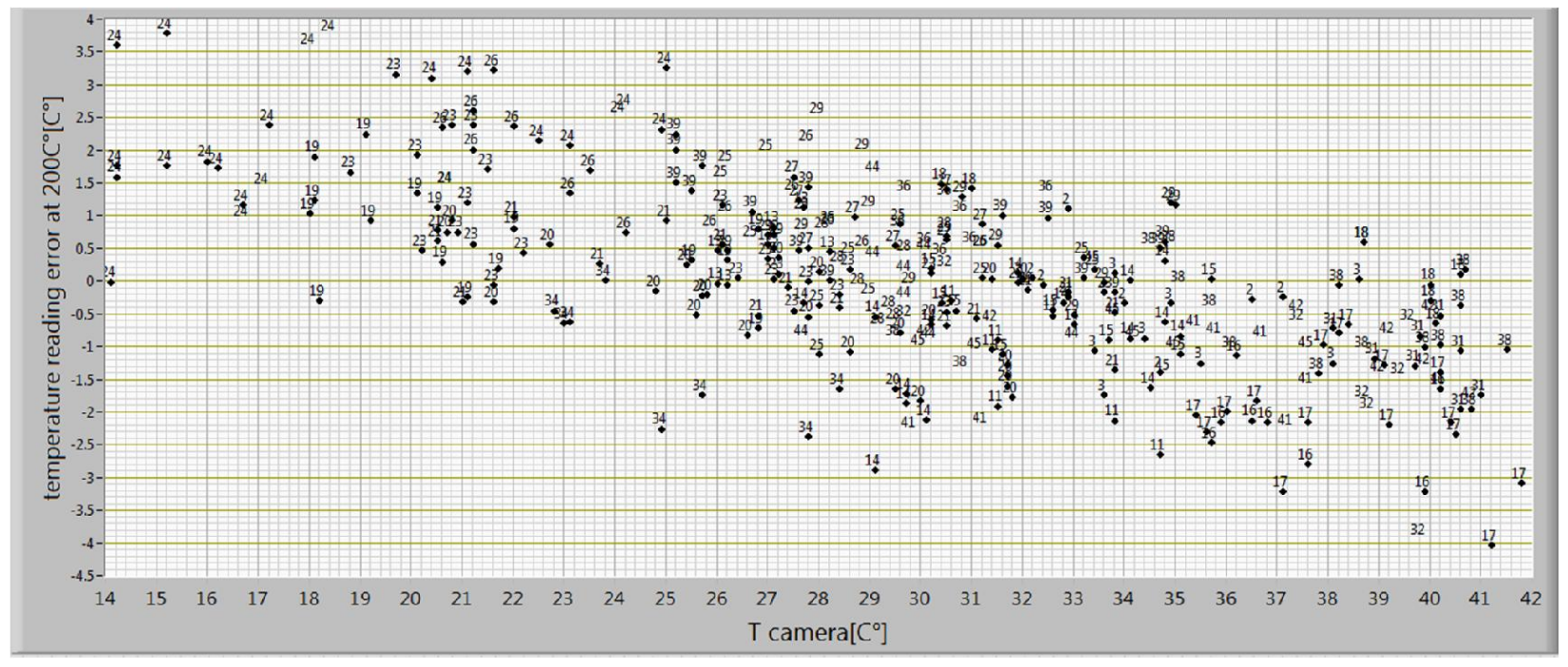

Fig 8. Empirical data of accuracy as function of camera temperature

The last error that was found, and compensated for, came from the Germanium window temperature. The Germanium used for the window has a $35 \mathrm{ohm}-\mathrm{cm}$ electrical resistivity. This window has a low transmission factor at room temperature of about 3\%; but the transmission decreases as the window's temperature increases [4], reaching $50 \%$ and higher transmission at $100 \mathrm{C}^{\circ}$ (wavelength dependent). The windows are exposed to both ambient temperature and, at some times of the day, even direct sun. Because of this, the temperature was unstable, and influenced the measurements' accuracy. A thermocouple was added on the windows, and its reading compensates for the transmission changes by the control software.

\section{Conclusion}

The IR cameras are a critical component in the control of the solar field in concentrated solar power fields. Due to specific conditions apparent in the concentrated solar power fields, it was necessary to develop a calibration method for the cameras. The calibration method revealed the different factors and their effects on the IR cameras readings. Both software and hardware solutions were implemented to minimize the accuracy uncertainty; and thus improve solar field performance. 


\section{REFERENCES}

[1] G. Naor, G. Goldwine, R. Hayut, O. Bibi, E. Silberstein, O. Chernin, Z. Auman, G. Kroyzer, and A. Ziskin, 2010, "Flux Measurement System Using IR Camera," in proceedings of SolarPACES 2010,Perpignan, France, September 21-24, 2010.

[2] Minkina, W. and Dudzik, S. (2009) Infrared Thermography: Errors and Uncertainties, John Wiley \& Sons, Ltd, Chichester, UK

[3] Vollmer, M. and Möllmann, K.-P. (2010) Infrared Thermal Imaging: Fundamentals, Research and Applications, Wiley-VCH Verlag GmbH \& Co. KGaA, Weinheim, Germany

[4] Jroy C. Richter ; Charles R. Poznich and Dennis W. Thomas "Minimization of IR absorption by germanium at elevated temperatures", Proc. SPIE 1326, Window and Dome Technologies and Materials II, 106 (October 1, 1990); doi:10.1117/12.22488 